\title{
EML4-ALK induces cellular senescence in mortal normal human cells and promotes anchorage- independent growth in hTERT-transduced normal human cells
}

\author{
Akihiko Miyanaga \\ National Instiutes of Health \\ Masaru Matsumoto \\ National Cancer Institute \\ Jessica A. Beck \\ National Cancer Institute \\ Izumi Horikawa \\ National Cancer Instiute \\ Takahiro Oike \\ National Cancer Instiute \\ Hirokazu Okayama \\ National Cancer Institute \\ Hiromi Tanaka \\ Indiana University of Medicine \\ Sandra S. Burkett \\ National Cancer Institute \\ Ana I. Robles \\ National Cancer Institute \\ Mohammed Khan \\ National Cancer Institute \\ Delphine Lissa \\ National Cancer Institute \\ Masahiro Seike \\ Nippon Medical School \\ Akihiko Gemma \\ Nippon Medical School
}

Hiroyuki Mano

National Cancer Reseach Insitute

Curtis C. Harris ( $\nabla$ harrisc@mail.nih.gov ) 
Research article

Keywords: EML4-ALK, lung cancer, senescence, anchorage-independent growth, hTERT, DNA damage

Posted Date: August 26th, 2020

DOI: https://doi.org/10.21203/rs.3.rs-35307/v2

License: (c) (i) This work is licensed under a Creative Commons Attribution 4.0 International License. Read Full License

Version of Record: A version of this preprint was published on March 24th, 2021. See the published version at https://doi.org/10.1186/s12885-021-07905-6. 


\section{Abstract}

Background: Chromosomal inversions involving anaplastic lymphoma kinase $(A L K)$ and echinoderm microtubule associated protein like 4 (EML4) generate a fusion protein EML4-ALK in non-small cell lung cancer (NSCLC). The understanding of EML4-ALK function can be improved by a functional study using normal human cells.

Methods: Here we for the first time conduct such study to examine the effects of EML4-ALK on cell proliferation, cellular senescence, DNA damage, gene expression profiles and transformed phenotypes.

Results: The lentiviral expression of EML4-ALK in mortal, normal human fibroblasts caused, through its constitutive ALK kinase activity, an early induction of cellular senescence with accumulated DNA damage, upregulation of $\mathrm{p} 16^{\mathrm{INK} 4 \mathrm{~A}}$ and $\mathrm{p} 21^{\mathrm{WAF} 1}$, and senescence-associated b-galactosidase (SA- $\beta$-gal) activity. In contrast, when EML4-ALK was expressed in normal human fibroblasts transduced with telomerase reverse transcriptase (hTERT), which is activated in the vast majority of NSCLC, the cells showed accelerated proliferation and acquired anchorage-independent growth ability in soft-agar medium, without accumulated DNA damage, chromosome aberration, nor p53 mutation. EML4-ALK induced the phosphorylation of STAT3 in both mortal and hTERT-transduced cells, but RNA sequencing analysis suggested that the different signaling pathways contributed to the different phenotypic outcomes in these cells. While EML4-ALK also induced anchorage-independent growth in hTERTimmortalized human bronchial epithelial cells in vitro, the expression of EML4-ALK alone did not cause detectable in vivo tumorigenicity in immunodeficient mice.

Conclusions: Our data indicate that the expression of hTERT is critical for EML4-ALK to manifest its in vitro transforming activity in human cells. This study provides the isogenic pairs of human cells with and without EML4-ALK expression.

\section{Background}

The ALK gene undergoes chromosomal translocations and fusions with other genes to generate oncogenic fusion proteins in non-small cell lung carcinoma (NSCLC) and other malignancies [1-3]. The $E M L 4$ gene is the most frequent fusion partner of $A L K$ in NSCLC, resulting in an oncogenic fusion protein EML4-ALK $[3,4]$. The tumors with EML4-ALK rarely have the other genetic changes such as mutations of EGFR, KRAS and TP53 [5], suggesting that their development strongly depends on the oncogenic fusion protein. The inhibition of the ALK tyrosine kinase activity has led to major advances in treatment of patients with ALK fusion-positive tumors $[2,6,7]$.

The basic understanding of the biological activities of ALK fusion proteins remains incomplete, partly due to the lack of functional studies using normal human cells. Previous studies used murine cell lines, human cancer-derived cell lines or chromosomally abnormal immortalized human cells [4, 8-10]. This study for the first time investigates the function of EML4-ALK in mortal, normal human cells, as well as in hTERT-transduced normal human cells. Our data show the different activities of EML4-ALK in mortal and 
hTERT-transduced normal human cells and provide mechanistic insight into its role during human carcinogenesis.

\section{Methods}

\section{Cells and cell culture}

CRL-2097, BJ and MRC-5 and NIH/3T3 were obtained from ATCC (Manassas, VA, USA) and maintained in DMEM supplemented with 10\% FBS. HBET1 [11] and their derived cells were maintained in LHC-9 medium (Thermo Fisher Scientific, Waltham, MA USA) supplemented with 2 mM L-glutamine. H3122 was obtained from the NCI Repository of Tumor Cell Lines (Frederick, MD, USA) and maintained in RPMI 1640 medium supplemented with $10 \%$ FBS. For tetracycline (Tet)-inducible gene expression, doxycycline (Dox, at $1 \mu \mathrm{g} / \mathrm{ml}$ ) was added. In continuous culture of human fibroblasts to examine cellular replicative lifespan, the cells were passaged at a split ratio of 1:4 (or 1:2 at later passages when approaching senescence). The number of population doubling levels (PDL) achieved between passages was determined by $\log _{2}$ ("number of cells obtained" divided by "number of cells inoculated") [11] and data were presented as means \pm s.d. from biological triplicates. Crizotinib was purchased from Selleck Chemicals (Houston, TX, USA) and used at a concentration of $25 \mathrm{nM}$. For all cells, culture medium was changed every 48 hours. For cells treated with Dox or Dox plus Crizotinib, they were continuously included in the medium.

SA- $\beta$-gal staining was performed using the kit purchased from Cell Signaling Technology (Danvers, MA, USA).

\section{Lentiviral and retroviral expression vectors and vector transduction}

pLenti3.3/TR and a lentiviral expression vector pLenti6.3/TO/V5-DEST were from Thermo Fisher Scientific. The EML4-ALK cDNA variant $1[3,4]$ was transferred into pLenti6.3/TO/V5-DEST for its inducible (with pLenti3.3/TR) and constitutive expression (without pLenti3.3/TR). The red fluorescent protein (RFP) CDNA was also transferred from pLOC (Open Biosystem, Lafayette, CO, USA) to pLenti6.3/TO/V5-DEST, generating the control vector. EML4-ALK (K589M) in pLenti6.3/TO/V5-DEST was generated via site-directed mutagenesis using the QuikChange II XL kit (Stratagene, Carlsbad, CA, USA). The retroviral expression vector for H-RasV12 (in pBabe vector) was a gift from Dr. Manuel Serrano (IRB Barcelona, Spain) [12]. The retroviral vector for hTERT (pCLXSN-hTERT) was previously described [11] and used to generate hTERT-immortalized BJ (hTERT-BJ) and HBET1 previously $[11,13]$ and hTERTtransduced CRL-2097 (hTERT-CRL-2097) in this study. The preparation of vector supernatants and the vector transduction were performed as previously described $[11,14,15]$. Two days after transduction, the cells were selected with puromycin (for pBabe, $1 \mathrm{mg} / \mathrm{ml}$; Sigma-Aldrich), G418 (for pLenti3.3/TR and pCLXSN-hTERT, $500 \mathrm{mg} / \mathrm{ml}$; Sigma-Aldrich) or blasticidin (for pLenti6.3/TO/V5-DEST, $2 \mathrm{mg} / \mathrm{ml}$; Thermo Fisher Scientific).

The coding sequences in all newly constructed vectors were fully sequenced for confirmation. 


\section{Protein lysates and western blot analysis}

Cells were lysed in 20 mM Tris- $\mathrm{HCl}(\mathrm{pH} 7.5)$ / $150 \mathrm{mM} \mathrm{NaCl} / 0.1 \%$ SDS / 1\% NP-40 / 1 mM EDTA containing the Complete protease inhibitor cocktail (Roche Diagnostics, Indianapolis, IN, USA). Western blot analysis was performed as described previously $[11,16]$. Signal detection was performed using the SuperSignal West Dura chemiluminescence substrate (Thermo Fisher Scientific) and the images were captured using the ChemiDoc Imager (Bio-Rad, Hercules, CA, USA). Quantitative image analysis used the ImageJ software (http://rsb.info.nih.gov/ij/). Primary antibodies used were as follows: anti-ALK (\#3633, Cell Signaling Technology; 1:2000); anti-phosho-ALK (\#3341, Cell Signaling Technology; 1:1000); antiSTAT3 (\#9139, Cell Signaling Technology; 1:1000); anti-phospho-STAT3 (\#9145, Cell Signaling Technology; 1:2000); anti-AKT (\#9272, Cell Signaling Technology; 1:1000); anti-phospho-AKT (\#9271, Cell Signaling Technology; 1:1000); anti-Src (\#2123, Cell Signaling Technology; 1:1000); anti-phospho-Src (\#6943, Cell Signaling Technology; 1:1000); anti-Erk1/2 (\#9102, Cell Signaling Technology; 1:1000); antiphosho-Erk1/2 (\#9101, Cell Signaling Technology; 1:1000); anti-c-H-Ras (\#OP23, Calbiochem; 1:30); antip16 ${ }^{\text {INK4A }}$ (sc-468, Santa Cruz Biotechnology, Dallas, TX, USA; 1:1000); anti-p21 WAF1 (\#2947, Cell Signaling Technology; 1:1000); anti-p53 (D0-1, sc-126, Santa Cruz Biotechnology, Dallas, TX, USA; 1:1000); antiphospho-p53 (Ser15) (\#9284, Cell Signaling Technology; 1:1000); and anti-GAPDH (sc-166574, Santa Cruz Biotechnology; 1:1000.

\section{Immunofluorescence staining}

Cells ( $3.0 \times 10^{4}$ cells/well) were washed with PBS and fixed for 10 min with $4 \%$ paraformaldehyde, followed by permeabilization with $0.25 \%$ Triton-X-100 for 10 min. Incubation with anti-g-H2AX antibody (Catalog \#05-636, Sigma Aldrich; 1:1000) and then with an Alexa Fluor 488-conjugated secondary antibody (Catalog \#A-21202; Thermo Fisher Scientific; 1:400), washing procedures, and mounting with Antifade with DAPI (Vectashield, Burlingame, California) were as previously described $[17,18]$. Digital images were acquired and analyzed using confocal microscopy (Zeiss 780) and ZEN software. The total signal intensity of $\mathrm{Y}-\mathrm{H} 2 \mathrm{AX}$ immunofluorescence per microscopic field was determined in unmerged $\mathrm{Y}^{-}$ $\mathrm{H} 2 \mathrm{AX}$ images using Image $\mathrm{J}$ software and expressed as the mean signal intensity per DAPI+ nucleus manually quantified in five high powered fields (40x).

\section{Anchorage-independent colony formation in soft-agar medium}

Cells $(1,000$ cells/well) were seeded on 12 -well plates in $1 \mathrm{ml}$ of medium containing $0.35 \%$ agarose (SeaPlaque low-melting-temperature agarose, Lonza Biosciences, Alpharetta, GA, USA) over 1-ml volume of base layer consisting of the same culture medium and $0.5 \%$ agarose. Colonies of approximately 50 $\mathrm{mm}$ in diameter or larger were counted on day 21.

\section{Telomere length measurement by quantitative PCR (qPCR)}

Telomere length was measured by the monochrome multiplex qPCR (MMQPCR) method as described previously [19]. Relative telomere length was express as a ratio of the quantity of telomeric DNA (T) 
normalized to the quantity of multiple copy sequence DNA (mcs), yielding T/M ratio. Intra- and interassay coefficient of variations (CVs) were $4.1 \%$ and $10.7 \%$, respectively.

\section{G-band karyotyping and spectral karyotyping (SKY)}

Treatment with Colcemid (10 mg/ml; KaryoMax, Invitrogen, Carlsbad, CA, USA), hypotonic treatment $(0.075 \mathrm{M} \mathrm{KCl})$, fixation with methanol/acetic acid (3:1), slide preparation and G-banding were performed as previously described [20] and the images were captured and analyzed with the HiBand system (Applied Spectral Imaging, Carlsbad, CA, USA). For spectral karyotyping (SKY), the slides were processed using the 24-color Human SKY Paint kit (Applied Spectral Imaging) according to the manufacturer's protocol. Spectral images of the hybridized metaphases were acquired using the HyperSpectral Imaging system (Applied Spectral Imaging) and analyzed using the HiSKY v.7.2 acquisition software (Applied Spectral Imaging). All cell lines were analyzed by G-band karyotyping. hTERT-CRL-2097, hTERT-CRL2097+EML4-ALK, its derived soft-agar clones \#1 and \#2, and HBET1 were also analyzed by SKY.

\section{Tumorigenicity in vivo}

Female NOD.SCID/Ncr mice were obtained from Charles River Laboratories (\#560; Germantown, MD, USA), and were maintained under specific pathogen-free conditions and the animals had free access to feedstuff and water under 12:12 light/dark cycles, 22 and $30-70 \%$ humidity. These mice at 6 to 10 weeks of age were subcutaneously injected with cells ( $5^{\prime} 10^{6}$ per flank) in 50\% Matrigel (no. 354248, Corning, NY, USA) at both flanks: one side with EML4-ALK-expressing cells or their derived soft-agar clones; and the other side with vector control cells. For injection of these cells, mice were anesthetized using isoflurane in accordance with $\mathrm{NCl}$ Animal Care and Use Committee (ACUC) guidelines. As a positive control, NIH/3T3 cells expressing EML4-ALK were injected at both flanks of two mice ( $2^{\prime} 10^{6}$ per flank). Tumor size and body weight were measured twice a week until 8 weeks or tumor size excess $20 \mathrm{~mm}$. To ameliorate the suffering of mice observed throughout experimental studies, mice were euthanized by $\mathrm{CO}_{2}$ inhalation. After 8 weeks of administration, the mice were sacrificed and photographed, and the tumors were removed and weighed. This experiment was approved by the NCI ACUC (LHC-012-D).

\section{RNA isolation, reverse transcription (RT)-PCR and Sanger sequencing of the entire coding region of p53 mRNA}

Total RNA isolation, reverse transcription, and 1st strand cDNA synthesis with random hexamers were carried out as previously described [11, 15]. PCR amplification of the entire coding region of p53 mRNA was carried out using the Platinum Taq DNA Polymerase High Fidelity (Thermo Fisher Scientific) with the primers 5'-ATG GAG GAG CCG CAG TCA-3' and 5'-GTC AGT CTG AGT CAG GCC CTT C-3'. The amplified PCR product was sequenced using the BigDye Terminator v1.1 Cycle Sequencing kit (Thermo Fisher Scientific) with 5'-AGT ACG TGC AAG TCA CAG-3', 5'-CGT CCC AAG CAA TGG ATG-3' and 5'-CTC ACC ATC ATC ACA CTG G-3'.

\section{RNA sequencing (RNA-seq) analysis}


RNA samples were treated with DNase I (no. 18068015, Thermo Fisher Scientific), followed by purification with RNeasy MinElute Cleanup kit (Qiagen). The RNA integrity numbers (RIN) of the samples on the Agilent 2100 Bioanalyzer (Agilent Technologies) were 9.3 to 10.0. All the following steps of the RNA-seq experiment were carried out at the CCR Sequencing Facility (Leidos Biomedical Research, Frederick, MD, USA). Sequencing libraries were prepared using the TruSeq Stranded mRNA Library Prep kit (Illumina, San Diego, CA, USA) and were sequenced with paired-end reads of $150 \mathrm{bp}$ on HiSeq3000/4000 sequencer (Illumina) to obtain at least 30 million read pairs per sample. Reads were trimmed for adaptors and lowquality bases using Trimmomatic [21] and were aligned with the reference genome (Human-hg38) and the annotated transcripts (Gencode_v24) using STAR (https://github.com/alexdobin/STAR). The gene expression quantification analysis was performed using STAR/RSEM tools (http://deweylab.github.io/RSEM/) to obtain raw read counts and normalized read counts (RPKM) for each gene.

Differentially expressed genes were identified using DESeq2 in $\mathrm{R}$ [22] with a false discovery rate (FDR) cutoff of 0.01 , and analyzed by gene enrichment analysis for KEGG (https://www.genome.jp/kegg/) and Reactome Pathways (https://reactome.org/) using DAVID v6.8 (https://david.ncifcrf.gov/). Enriched pathways were identified according to FDR $£ 0.05$.

\section{MicroRNA quantification by qPCR}

PCR was performed by TaqMan MicroRNA Assays as manufacturer's instructions. Primers used were as follows: miR-21 (Taqman microRNA assay, hsa-miR-21, PN 4440887); RNU48 (Taqman microRNA assay, RNU48, PN 4440887).

\section{Data mining of publicly available lung adenocarcinoma datasets}

For the TCGA dataset [23], mRNA expression from RNA Seq V2 RSEM and tumor characteristics were downloaded from cBioPortal for 510 patients with lung adenocarcinoma. Log2-transformed RSEM values for hTERT were used for the analyses.

For the microarray dataset GSE31210 [24], data and sample characteristics were downloaded from the Gene Expression Omnibus for 226 patients with lung adenocarcinoma using the R package GEOquery. The raw intensity values were processed and normalized with Robust Multi-Array Average (RMA) using the R package oligo. Affymetrix IDs were mapped to HUGO Gene Nomenclature Committee IDs. Resulting RMA expression values for hTERT were used for the analyses.

\section{Quantitative RT-PCR analysis}

Quantitative RT-PCR analysis to confirm the RNA-seq data was performed by using TaqMan Assay as manufacture's instruction. Primer used was as follows: IL1B (Taqman Hs01555410_m1, Cat 4331182), CXCL8 (Taqman Hs00174103_m1, Cat 4331182), GAPDH (Taqman Hs02786624_g1, Cat 4331182), PLAU (Taqman Hs01547050_m1, Cat 4331182), PLAT (Taqman Hs 00938315_m1, Cat 4331182) and A2M (Taqman Hs 00929971_m1, Cat 4331182). 


\section{Statistical analysis}

Data are presented as means \pm s.d. from at least three biological replicates. Statistical significance was evaluated using unpaired 2-tailed Student's $t$-test. * $P<0.05$, ** $P<0.01$ and $* \star * ~ P<0.001$.

\section{Results}

\section{EML4-ALK causes the early induction of cellular senescence in normal, mortal human fibroblasts}

We transduced normal human fibroblasts CRL-2097, which are mortal, or have a limited replicative lifespan, with a lentiviral vector expressing the Tet repressor (generating CRL-2097/TR), and then with either a lentiviral vector encoding EML4-ALK or the control vector (Fig. 1a). While the control cells with no EML4-ALK expression reached approximately 11 or 12 population doubling levels (PDL) before proliferation arrest, the EML4-ALK-expressing cells ceased to proliferate earlier at PDL 6 after lentiviral transduction (Fig. 1b). Both the control and EML4-ALK-expressing cells, upon proliferation arrest, were similarly positive for SA- $\beta$-gal (Fig. 1C). In addition to the cells shown in Fig. 1a (observed through day 88 or 40 in Fig. 1b), those retrovirally transduced with oncogenic Ras (H-RasV12) and its control vector (pBabe) were examined (Supplementary Fig. S1A; observed through day 20 in Fig. 1b) and H-RasV12induced senescence was observed (Supplementary Fig. S1B). Thus, the early induction of cellular senescence by EML4-ALK was reminiscent of oncogenic Ras-induced senescence [12], although $\mathrm{H}$ RasV12 induced-senescence occurred earlier in this CRL-2097 strain (Fig. 1b, Supplementary Fig. S1B). The high level of EML4-ALK expression might have been selected against during entry into senescence (Dox+ at PDL 6 in Fig. 1a; also see below Discussion).

\section{p16 ${ }^{\text {INK4A }}$ and p21 ${ }^{\text {WAF1 }}$ are upregulated in both natural replicative senescence and EML4-ALK-induced early senescence}

The expression levels of $p 16^{\text {INK4A }}$ and $p 21^{\mathrm{WAF} 1}$, two markers of cellular senescence $[25,26]$, were examined in CRL-2097/TR with EML4-ALK or H-RasV12 expression, along with their control cells (Fig. 1d). The $16^{\text {INK4A }}$ protein expression was increased during the replicative lifespan in both EML4-ALKexpressing and control cells, although its levels at senescence were lower than observed in H-RasV12induced senescence. An increase in p21 ${ }^{\text {WAF1 }}$ protein expression was also observed similarly in the EML4ALK-expressing and control cells, and to a lesser degree in H-RasV12-induced senescence. These findings suggest that the similar levels of upregulation of $\mathrm{p} 16^{\mathrm{INK} 4 \mathrm{~A}}$ and $\mathrm{p} 21^{\mathrm{WAF} 1}$ occur with the EML4-ALKinduced senescence and natural replicative senescence, although the former undergoes fewer PDL than the latter to achieve those expression levels.

\section{The EML4-ALK-induced senescence is associated with accumulated DNA damage}

A phosphorylated H2AX (g-H2AX) [13] was detected by immunofluorescence staining in CRL-2097/TR fibroblasts with EML4-ALK expression (EML4-ALK-Dox+ in Fig. 1b) and with the control vector (Vector in Fig. 1b) when approaching senescent proliferation arrest (at PDL 6 and PDL 12, respectively) (Fig. 1e-f). 
The early induction of cellular senescence in the EML4-ALK-expressing cells was associated with significantly more accumulation of $\mathrm{g}-\mathrm{H} 2 \mathrm{AX}$ foci (Fig. 1e-f), suggesting that EML4-ALK-induced senescence accompanies an accelerated rate of persistent DNA damage per PDL compared with natural replicative senescence. The level of p53 protein phosphorylated at serine 15, a target residue of the ATM kinase signaling from DNA damage to cellular senescence [27-29], was increased in both control and EML4-ALK-expressing cells upon senescence (Supplementary Fig. S2A).

A quantitative PCR-based measurement of telomere length showed that the vector control cells underwent progressive telomere shortening through their replicative senescence at PDL 12 (Supplementary Fig. S2B). The EML4-ALK-expressing cells had the telomere length shorter than that of the original cells before lentiviral transduction, similar to that of the control cells at a comparable PDL (PDL 7) and longer than that of the replicatively senescent control cells at PDL 12 (Supplementary Fig. $\mathrm{S} 2 \mathrm{~B})$. These data suggest that normal, mortal human fibroblasts with and without EML4-ALK expression undergo telomere shortening at similar rates per PDL, and that the accelerated accumulation of DNA damage in the EML4-ALK-expressing cells may be of non-telomeric origin [13].

\section{The EML4-ALK-induced senescence depends on its ALK kinase activity}

The EML4-ALK-expressing CRL-2097/TR with Dox addition were treated with an ALK tyrosine kinase inhibitor (TKI) Crizotinib at $25 \mathrm{nM}$ (a concentration close to the reported $\mathrm{IC}_{50}$ value [30]) and were monitored for cell proliferation. The treatment with Crizotinib inhibited the autophosphorylation of EML4ALK while not affecting the expression level of EML4-ALK, confirming its TKI activity against the ALK kinase activity (Fig. 2a). The expression of EML4-ALK without Crizotinib reproducibly induced the proliferation arrest at PDL 6 (Fig. 2b). In contrast, the Crizotinib-treated cells bypassed this early proliferation arrest and underwent approximately 4 more PDL, similarly to the control cells without EML4ALK expression (Fig. 2b).

Another normal human fibroblast strain MRC-5 (without a Tet repressor) was transduced with the lentiviral vector encoding wild-type EML4-ALK or a kinase-dead mutant of EML4-ALK (K589M) [4], along with the control vector. The K589M mutant of EML4-ALK was constitutively expressed at a higher level than the wild-type counterpart but was not autophosphorylated (Fig. 2c). Since the MRC-5 fibroblasts used in this experiment were closer to natural replicative senescence than the CRL-2097 fibroblasts used above, the control vector-transduced cells underwent at most 5 PDL before they ceased to proliferate (Fig. 2d). The expression of wild-type EML4-ALK, again in these fibroblasts approaching natural replicative senescence, caused earlier induction of proliferation arrest (Fig. 2d) with SA-b-gal (Fig. 2e), which was similar to oncogenic Ras-induced senescence (Supplementary Fig. S1C-D). Importantly, the K589M mutant-expressing cells did not show early proliferation arrest and behaved like the control vectortransduced cells (Fig. 2d-e), further supporting that the ALK kinase activity mediates the early induction of cellular senescence by EML4-ALK.

EML4-ALK promotes anchorage-independent growth in hTERT-transduced normal human fibroblasts 
Although EML4-ALK-positive NSCLC rarely have other accompanying genetic alterations [5], they still have a mechanism to maintain telomere length and function, in most cases via hTERT activation [31]. Consistent with the previous findings [32], ALK fusion-positive NSCLC tumor tissues, as well as negative ones, were confirmed to express hTERT (Supplementary Fig. S3A and B). We thus hypothesized that EML4-ALK might cooperate with the expression of hTERT, leading to telomerase activation and cell immortalization, to cause cellular transformation in normal human cells. To test this hypothesis, hTERT was retrovirally transduced into CRL-2097 to establish an hTERT-transduced normal human cell line (hTERT-CRL-2097), which maintained elongated telomeres (Supplementary Fig. S4A). This cell line had normal karyotype (Supplementary Fig. S4B), maintained normal p16 ${ }^{\text {INK4A }}$ response to oncogenic Ras (Supplementary Fig. S4C) and retained wild-type TP53 (below in Fig. 3e), thus not coincident with the changes frequently associated with human cell immortalization. These hTERT-CRL-2097 cells were transduced with the wild-type EML4-ALK vector or the control vector, and the constitutive expression and autophosphorylation of the EML4-ALK protein was confirmed (Fig. 3a). Unlike in mortal CRL-2097, the expression of EML4-ALK in this hTERT-transduced cell line did not induce senescent proliferation arrest, but instead resulted in accelerated cell proliferation (Fig. 3b). The hTERT-CRL-2097 cells, with or without EML4-ALK expression, accumulated no or little DNA damage (g-H2AX foci in Fig. 3c and Supplementary Fig. S5), consistent with no induction of cellular senescence and in contrast to DNA damage accumulation in mortal CRL-2097 fibroblasts (the leftmost bar in Fig. 3c; and Fig. 1e above). Furthermore, the anchorage-independent formation of cell colonies in soft-agar medium was enhanced in the EML4ALK-expressing hTERT-CRL-2097 cells, which was inhibited by treatment with the ALK TKI Crizotinib (Fig. $3 d)$.

In another hTERT-transduced normal human fibroblast cell line, hTERT-BJ (Supplementary Fig. S6A), the expression of wild-type EML4-ALK, but not of the K589M mutant, accelerated cell proliferation (Supplementary Fig. S6B) and promoted anchorage-independent growth in soft agar (Supplementary Fig. $\mathrm{S} 6 \mathrm{C})$. The ability of wild-type EML4-ALK to promote anchorage-independent growth was again abrogated by treatment with the ALK TKI Crizotinib (Supplementary Fig. S6C). From our reproducible results in two lines of hTERT-transduced normal human fibroblasts, we conclude that EML4-ALK has in vitro transformation activity in these cells through its constitutive ALK kinase activity.

\section{The EML4-ALK-induced anchorage-independent growth occurs without chromosome aberrations and without loss or mutation of the TP53 gene}

We performed karyotype analysis in hTERT-CRL-2097 cells transduced with the control vector, those expressing EML4-ALK and six of their derived clones isolated from soft-agar culture. All of these cells examined, as well as the original hTERT-CRL-2097 cells (as mentioned above and Supplementary Fig. S4B), maintained normal male karyotype 46, XY (Fig. 3e and Supplementary Fig. S7A-B). All cell lines listed in Fig. 3e had normal male karyotype 46, XY by G-banding (at least 10 well-spread metaphases per line were examined; an example is shown in Supplementary Fig. S7A). hTERT-CRL-2097, hTERT-CRL2097+EML4-ALK and soft-agar clones \#1 and \#2 were also confirmed by SKY to have 46, XY (again, at least 10 metaphases per line were examined; an example is shown in Supplementary Fig. S7B). By direct 
sequencing of the RT-PCR products, the entire coding region of TP53 was shown to be wild-type without a clonal homozygous or heterozygous mutation. The polymorphic codon 72 of TP53 was heterozygous (Pro/Arg) in the original hTERT-CRL-2097 and remained heterozygous (Pro/Arg) in hTERT-CRL-2097 expressing EML4-ALK and their derived soft-agar clones (examples are shown in Supplementary Fig. S7C), indicating that no chromosome instability nor loss of heterozygosity ( $\mathrm{LOH}$ ) occurred at TP53 during EML4-ALK-induced acquisition of anchorage-independent growth.

Although loss or mutation of the TP53 gene is frequently associated with cell transformation and carcinogenesis [33], all of the EML4-ALK-expressing cells and their derived soft-agar clones had the wildtype TP53 sequence without any homozygous or heterozygous mutation (Fig. 3e). The original hTERTCRL-2097 cells showed Pro/Arg heterozygosity at the polymorphic codon 72, which was maintained in the EML4-ALK-expressing cells and all the soft-agar clones (Fig. 3e and Supplementary Fig. S7C), indicating that there was no loss of a TP53 allele in these cells. The p16 ${ }^{\text {INK4A }}$ pathway, which is frequently impaired during cell transformation and carcinogenesis [26, 34], was suggested to remain intact during the EML4-ALK-mediated cell transformation by the finding that oncogenic Ras-induced p16 $6^{\text {INK4A }}$ upregulation [12] was observed in the EML4-ALK-expressing cells (Supplementary Fig. S7D).

\section{STAT3 is phosphorylated by EML4-ALK in both mortal and hTERT-transduced normal human fibroblasts}

We examined the activation status of three major factors downstream of EML4-ALK (i.e., STAT3, Akt and Erk1/2) by western blot (Fig. 4a and 4b). Mortal normal human fibroblasts CRL-2097/TR with and without EML4-ALK expression both showed increased levels of phosphorylated Akt when they became senescent, while the levels of phosphorylated Erk1/2 did not show a consistent change associated with EML4-ALK expression or increased PDL levels (Fig. 4a). Notably, a striking induction of STAT3 phosphorylation was observed in the EML4-ALK-expressing cells at PDL 4, which was decreased upon senescent proliferation arrest (PDL 6) but still at a higher level than that in the control cells at senescence (PDL 11) (Fig. 4a). Also in hTERT-transduced CRL-2097 fibroblasts, the expression of EML4-ALK resulted in remarkable induction of phosphorylated STAT3, while no increase in phosphorylated Akt and a slight increase in phosphorylated Erk1/2 were associated with EML4-ALK expression (Fig. 4b). These findings suggest that STAT3 functions as a downstream effector of EML4-ALK in both mortal and hTERTtransduced normal human fibroblasts, consistent with its involvement in both cellular senescence and transformation [35-38] and the previous studies directly linking EML4-ALK to STAT3 phosphorylation [39, 40]. MiR-21, a microRNA induced by STAT3 [41], was also upregulated by EML4-ALK in both mortal and hTERT-transduced fibroblasts (Supplementary Fig. S8). Although a decrease in phosphorylated Src was associated with cellular senescence in the presence or absence of EML4-ALK (Fig. 4a), the expression of EML4-ALK did not affect the phosphorylation level of Src (Fig. 4a and 4b), which is known to mediate acquired resistance to ALK TKIs [42].

\section{EML4-ALK regulates different signaling pathways in mortal and hTERT-transducednormal human fibroblasts}


We performed RNA sequencing (RNA-seq) in duplicated samples of mortal CRL-2097/TR with and without EML4-ALK expression (Supplementary Fig. S9A; Supplementary Table S1) and hTERT-transduced CRL-2097 with and without EML4-ALK expression (Supplementary Fig. S9B; Supplementary Table S2). The analysis of the differentially expressed genes to KEGG (Kyoto Encyclopedia of Genes and Genomes) Pathways (https://www.genome.jp/kegg/) identified the cytokine-cytokine receptor interaction pathway as significantly upregulated by EML4-ALK expression in mortal CRL-2097/TR fibroblasts, along with some related and overlapping pathways involving tumor necrosis factor (TNF) or viral infection (Table 1 and Supplementary Table S3). The interferon (IFN)-a/b and IFN-g signaling pathways, which have some genes in common with the above KEGG pathways, were also identified from Reactome Pathway Database (https://reactome.org/) as EML4-ALK-upregulated pathways in mortal CRL-2097/TR fibroblasts (Table 1 and Supplementary Table S3). Consistent with the EML4-ALK-induced phosphorylation of STAT3 (as above in Fig. 4), these cytokines and IFN signaling pathways include several STAT3-upregulated genes such as IL1B, CXCL8, SOCS3, IRF1 and IRF7 (Table 1 and Supplementary Table S3). The upregulation of IL1B and CXCL8 by EML4-ALK was confirmed by qRT-PCR (Supplementary Fig. S10). These data suggest that STAT3 may mediate the effect of EML4-ALK on activating the proinflammatory cytokine and IFN signaling cascades, which coordinately induce and maintain sustained DNA damage and senescent proliferative arrest in mortal normal human cells $[38,43]$.

In hTERT-transduced CRL-2097 fibroblasts, instead of the above-mentioned cytokine and IFN signaling pathways, the complement and blood coagulation cascades signaling was identified as significantly upregulated by EML4-ALK expression (Table 1 and Supplementary Table S3). This pathway included A2M, PLAT and PLAU as STAT3-upregulated genes $[44,45]$ (the upregulation of these genes was confirmed by qRT-PCR, Supplementary Fig. S11), suggesting that STAT3 may also mediate the modulation by EML4-ALK of blood coagulation, which may have clinical implications in increased risk of disseminated intravenous coagulation in patients with EML4-ALK-positive cancer [46]. Consistently, a Japanese cohort also showed an upregulation of the blood coagulation pathway in EML4-ALK-positive lung cancer [24] (Supplementary Fig. S12). We also found that some integrin and non-integrin components of focal adhesion and extracellular matrix (ECM) interactions were downregulated by EML4ALK in hTERT-transduced CRL-2097 fibroblasts (Table 1 and Supplementary Table S3), which likely contributed to EML4-ALK-induced anchorage-independent growth via overcoming anoikis [47, 48].

\section{EML4-ALK also has in vitro transforming activity in hTERT-immortalized normal human bronchial epithelial cells but does not cause in vivotumorigenicity}

The transforming activity of EML4-ALK was also tested in hTERT-immortalized, normal human bronchial epithelial cells, which represent a cell type more relevant to NSCLC pathogenesis. For this purpose, we used a previously established cell line, HBET1, which has a tetraploid karyotype with no or few structurally abnormal chromosomes (Supplementary Fig. S13), maintains elongated telomeres and does not show anchorage-independent growth or in vivo tumorigenicity [14]. The HBET1 cells constitutively expressing EML4-ALK with its autophosphorylation (Fig. 5a) showed accelerated cell proliferation (Fig. 5b) and acquired anchorage-independent growth in soft agar (Fig. 5c), as observed above in hTERT- 
transduced fibroblasts. The increased levels of phosphorylation of STAT3 and Akt, but not of Erk1/2 or Src, were associated with EML4-ALK expression in HBET1 cells (Fig. 5a). Like in hTERT-transduced CRL2097, PLAU and PLAT were upregulated by EML4-ALK in HBET1 cells as well (Supplementary Fig. S14).

To examine in vivo tumorigenicity, we injected HBET1 and hTERT-transduced CRL-2097 cells with and without EML4-ALK, along with the soft-agar clones derived from the EML4-ALK-expressing cells, subcutaneously into immunodeficient NOD.SCID/Ncr mice (Fig. $5 \mathrm{~d}$ ). None of these cells were able to form a growing tumor, while EML4-ALK-expressing mouse NIH/3T3 cells as a positive control [4] consistently formed progressively growing tumors (Fig. 5d). These results suggest that the expression of EML4-ALK alone is not sufficient for hTERT-transduced normal human cells to acquire in vivo tumorigenicity under our experimental conditions.

\section{Discussion}

This study for the first time performed the functional assays of an ALK fusion protein using normal human cells, which were mortal or hTERT-transduced. The major findings include: i) EML4-ALK, through its constitutive ALK kinase activity, induces mortal cells to undergo early senescence (Fig. 1 and 2); ii) EML4-ALK promotes hTERT-transduced cells to proliferate anchorage-independently (Fig. 3 and 5); iii) Accumulation of DNA damage in mortal but not hTERT-transduced cells (Fig. 1e and $3 \mathrm{c}$ ) is associated with senescence induction; and iv) EML4-ALK regulates the different signaling pathways in mortal and hTERT-transduced cells (Table 1).

In mortal normal human fibroblasts, the expression of EML4-ALK caused senescent proliferation arrest earlier than natural replicative senescence, but later than senescence induced by oncogenic Ras (Fig. 1b and Supplementary Fig. S1), which was previously reported to cause severer DNA damage [12, 49]. The EML4-ALK expression is likely to cause DNA damage (indicated by g-H2AX foci, Fig. 1e and $1 \mathrm{f}$ ) through oncogene-induced DNA hyper-replication and DNA replication stress [50, 51], leading to the ATM-mediated p53 activation [27-29] (indicated by its serine 15 phosphorylation, Supplementary Fig. S2A) and then to upregulation of a p53-activated senescence inducer p2 ${ }^{\text {WAF1 }}$ [25] (Fig. 1d), as well as leading to DNA damage-associated upregulation of $\mathrm{p} 16^{\mathrm{INK} 4 \mathrm{~A}}[12,52]$ (Fig. 1d). It is interesting to note a possible correlation between the severity of DNA damage and the degree of $\mathrm{p} 16^{\mathrm{INK} 4 \mathrm{~A}}$ upregulation in Ras-induced, EML4-ALK-induced and natural replicative senescence in this order (Fig. 1d).

A later phase of replicative lifespan of EML4-ALK-expressing mortal fibroblasts seemed to have selected against the cells with its higher expression level (compare Dox+ at PDL 4 and 6 in Fig. 1a), and thus the cells with its lower expression level likely underwent cell proliferation prior to complete proliferation arrest. Interestingly, the kinase-dead mutant K589M showed a higher expression level than wild-type EML4-ALK in mortal fibroblasts (Fig. 2c) and hTERT-transduced fibroblasts continued to maintain high levels of wildtype EML4-ALK expression during expansion (Fig. 3a). These findings suggest that reduced expression of wild-type EML4-ALK and its phosphorylated form upon senescence of mortal fibroblasts most likely 
reflects its kinase-dependent senescence-inducing activity, rather than due to technical issues leading to failed maintenance of expression.

Our findings highlight a significant contrast of EML4-ALK activities between mortal and hTERTtransduced normal human cells. Since hTERT and telomerase are suggested to contribute to genome stability not only at telomeres but also at non-telomeric sites [53, 54], hTERT-transduced cells may become less vulnerable to telomeric and non-telomeric DNA damage, leading to a functional switch of EML4-ALK from senescence induction to in vitro transformation. Consistently, while the expression of EML4-ALK in mortal normal human fibroblasts activated the STAT3-mediated signaling to DNA damage and senescence involving proinflammatory cytokines and IFN pathways [38, 43] (Table 1 and Supplementary Table S3), these pathways were not activated in EML4-ALK-expressing hTERT-transduced fibroblasts, which instead showed changes in gene expression that could contribute to their anchorageindependent growth (Table 1 and Supplementary Table S3).

Chromosome abnormality, loss or mutation of TP53, or impairment of p16 ${ }^{\text {INK4A }}$ response was not associated with EML4-ALK-induced anchorage-independent growth (Fig. 3e and Supplementary Fig. S7). These data are consistent with a previous finding that no or few chromosomal changes, other than an ALK-rearranging translocation, were observed in ALK fusion-positive tumors [1] and may reflect the molecular features of ALK fusion-positive tumors infrequently accompanying a genetic change affecting normal p53 and p16 INK4A functions [5]. Nonetheless, we speculate that other genetic or epigenetic events may also be involved in anchorage-independent growth induced by EML4-ALK. The higher efficiency of soft-agar colony formation in EML4-ALK-expressing HBET1 cells (Fig. 5c), compared with the counterparts of fibroblastic origin (Fig. 3d and Supplementary Fig. S6C), might be due to a genetic change(s) associated with numerical or structural chromosome aberrations that pre-existed in the original HBET1 cells (Supplementary Fig. S13) and/or due to hTERT immortalization-associated, pre-existing genetic and epigenetic changes frequently observed in epithelial cells [55] but not in fibroblasts [56].

Despite their ability of anchorage-independent growth, the EML4-ALK-expressing, hTERT-transduced normal human cells of fibroblastic origin (hTERT-CRL-2097) and of bronchial epithelial origin (HBET1) did not form tumors in immunodeficient mice under our experimental conditions (Fig. 5d), while mouse $\mathrm{NIH} / 3 \mathrm{~T} 3$ cells expressing EML4-ALK were highly tumorigenic [4]. This contrast further highlights the importance of this study using normal human, and strongly suggests that additional genetic and/or epigenetic changes are required for development of EML4-ALK-positive tumors, prompting us to identify a factor(s) or event(s) that cooperates with EML4-ALK for acquisition of fully malignant phenotypes.

Among candidate co-operating events to be examined is the inactivation of SETD2, a histone methyltransferase recently reported to be mutated in EML4-ALK fusion-driven lung adenocarcinoma [57].

\section{Conclusions}

Our data indicate that the expression of hTERT is critical for EML4-ALK to manifest its in vitro transforming activity in human cells. Out data also suggest that STAT3 is a downstream factor of EML4- 
ALK and the different signaling pathways contributed to the different phenotypic outcomes in normal and hTERT-transduced cells. In addition, this study has established the isogenic pairs of human cell lines with and without EML4-ALK expression. We expect that these isogenic cells will be a novel in vitro cell system for screening and testing new drug candidates and investigating drug resistance, with significant implications in translational and clinical medicine towards better treatment of ALK fusion-induced human malignancy.

\section{Declarations}

\section{Ethics approval and consent to participate}

The animal experiment was approved by the NCl Animal Care and Use Committee (LHC-012-D).

\section{Consent for publication}

Not Applicable.

\section{Availability of data and material}

All data generated and analyzed in this study are included in this published article and its supplementary information files. Raw data and materials are available from the corresponding author upon request.

\section{Competing interests}

The authors have no conflicts of interest to declare.

\section{Funding}

This research was supported by the Intramural Research Program of the $\mathrm{NIH}$, National Cancer Institute, Center for Cancer Research. The funding body was not involved in the design of the study and collection, analysis, and interpretation of data and in writing the manuscript.

\section{Author contributions}

$\mathrm{AM}, \mathrm{IH}$ and $\mathrm{CCH}$ conceived and designed the study; $\mathrm{AM}, \mathrm{MM}, \mathrm{JB}$, and $\mathrm{IH}$ performed the experiments. TO, $\mathrm{HO}, \mathrm{HT}, \mathrm{SB}, \mathrm{AR}, \mathrm{MK}$ and DL contributed data analysis. MS, AG and HM provided essential materials and expertise. $\mathrm{AM}, \mathrm{MM}$ and $\mathrm{IH}$ wrote the manuscript. $\mathrm{CCH}$ was responsible for the overall project. All authors provided critical revision and approved the final manuscript.

\section{Acknowledgments}

We thank CCR Genomics Core, CCR Sequencing Facility, Devorah Gallardo (Leidos Biomedical Research), Elisa Spillare, Elise Bowman and Teruhide Ishigame. 


\section{Abbreviations}

ALK, anaplastic lymphoma kinase; EML4, echinoderm microtubule associated protein like 4; NSCLC, nonsmall cell lung carcinoma; PDL, population doubling levels; hTERT, human telomerase reverse transcriptase; TKI(s), tyrosine kinase inhibitor(s); SA- $\beta$-gal, senescence-associated b-galactosidase; IFN, interferon

\section{References}

1. Rottgers S, Gombert M, Teigler-Schlegel A, Busch K, Gamerdinger U, Slany R et al: ALK fusion genes in children with atypical myeloproliferative leukemia. Leukemia 2010, 24(6):1197-1200.

2. Steuer CE, Ramalingam SS: ALK-positive non-small cell lung cancer: mechanisms of resistance and emerging treatment options. Cancer 2014, 120(16):2392-2402.

3. Takeuchi K, Soda M, Togashi Y, Suzuki R, Sakata S, Hatano S et al: RET, ROS1 and ALK fusions in lung cancer. Nat Med 2012, 18(3):378-381.

4. Soda M, Choi YL, Enomoto M, Takada S, Yamashita Y, Ishikawa $S$ et al: Identification of the transforming EML4-ALK fusion gene in non-small-cell lung cancer. Nature 2007, 448(7153):561-566.

5. Saito M, Shimada Y, Shiraishi K, Sakamoto H, Tsuta K, Totsuka H et al: Development of lung adenocarcinomas with exclusive dependence on oncogene fusions. Cancer Res 2015, 75(11):22642271.

6. Hallberg B, Palmer RH: Mechanistic insight into ALK receptor tyrosine kinase in human cancer biology. Nat Rev Cancer 2013, 13(10):685-700.

7. Sun Y, Nowak KA, Zaorsky NG, Winchester CL, Dalal K, Giacalone NJ et al: ALK inhibitor PF02341066 (crizotinib) increases sensitivity to radiation in non-small cell lung cancer expressing EML4-ALK. Mol Cancer Ther 2013, 12(5):696-704.

8. Lu H, Villafane N, Dogruluk T, Grzeskowiak CL, Kong K, Tsang YH et al: Engineering and Functional Characterization of Fusion Genes Identifies Novel Oncogenic Drivers of Cancer. Cancer Res 2017, 77(13):3502-3512.

9. Soule HD, Maloney TM, Wolman SR, Peterson WD, Jr., Brenz R, McGrath CM et al: Isolation and characterization of a spontaneously immortalized human breast epithelial cell line, MCF-10. Cancer Res 1990, 50(18):6075-6086.

10. Warmuth M, Kim S, Gu XJ, Xia G, Adrian F: Ba/F3 cells and their use in kinase drug discovery. Curr Opin Oncol 2007, 19(1):55-60.

11. Fujita K, Mondal AM, Horikawa I, Nguyen GH, Kumamoto K, Sohn JJ et al: p53 isoforms $\Delta 133$ p53 and p53beta are endogenous regulators of replicative cellular senescence. Nat Cell Biol 2009, 11(9):1135-1142.

12. Serrano M, Lin AW, McCurrach ME, Beach D, Lowe SW: Oncogenic ras provokes premature cell senescence associated with accumulation of p53 and p16INK4a. Cell 1997, 88(5):593-602. 
13. Nakamura AJ, Chiang YJ, Hathcock KS, Horikawa I, Sedelnikova OA, Hodes RJ et al: Both telomeric and non-telomeric DNA damage are determinants of mammalian cellular senescence. EpigeneticsChromatin 2008, 1:6.

14. Pine SR, Ryan BM, Varticovski L, Robles Al, Harris CC: Microenvironmental modulation of asymmetric cell division in human lung cancer cells. Proc Natl Acad Sci U S A 2010, 107(5):2195-2200.

15. Horikawa I, Fujita K, Jenkins LM, Hiyoshi Y, Mondal AM, Vojtesek B et al: Autophagic degradation of the inhibitory p53 isoform $\Delta 133 p 53$ alpha as a regulatory mechanism for p53-mediated senescence. Nat Commun 2014, 5:4706.

16. Horikawa I, Park KY, Isogaya K, Hiyoshi Y, Li H, Anami K et al: $\Delta$ 133p53 represses p53-inducible senescence genes and enhances the generation of human induced pluripotent stem cells. Cell Death Differ 2017, 24(6):1017-1028.

17. Turnquist C, Horikawa I, Foran E, Major EO, Vojtesek B, Lane DP et al: p53 isoforms regulate astrocyte-mediated neuroprotection and neurodegeneration. Cell Death Differ 2016, 23(9):1515-1528.

18. Turnquist C, Beck JA, Horikawa I, Obiorah IE, Von Muhlinen N, Vojtesek B et al: Radiation-induced astrocyte senescence is rescued by Delta133p53. Neuro Oncol 2019, 21(4):474-485.

19. Dahlgren PN, Bishop K, Dey S, Herbert BS, Tanaka H: Development of a New Monochrome Multiplex qPCR Method for Relative Telomere Length Measurement in Cancer. Neoplasia 2018, 20(5):425-431.

20. Schreck RR, Disteche CM: Chromosome banding techniques. Curr Protoc Hum Genet 2001, Chapter 4:Unit4.2.

21. Bolger AM, Lohse M, Usadel B: Trimmomatic: a flexible trimmer for Illumina sequence data. Bioinformatics 2014, 30(15):2114-2120.

22. Love MI, Huber W, Anders S: Moderated estimation of fold change and dispersion for RNA-seq data with DESeq2. Genome Biol 2014, 15(12):550.

23. Hoadley KA, Yau C, Hinoue T, Wolf DM, Lazar AJ, Drill E et al: Cell-of-Origin Patterns Dominate the Molecular Classification of 10,000 Tumors from 33 Types of Cancer. Cell 2018, 173(2):291-304.

24. Okayama H, Kohno T, Ishii Y, Shimada Y, Shiraishi K, Iwakawa R et al: Identification of genes upregulated in ALK-positive and EGFR/KRAS/ALK-negative lung adenocarcinomas. Cancer Res 2012, 72(1):100-111.

25. Brown JP, Wei W, Sedivy JM: Bypass of senescence after disruption of p21CIP1/WAF1 gene in normal diploid human fibroblasts. Science 1997, 277(5327):831-834.

26. Sharpless NE, DePinho RA: The INK4A/ARF locus and its two gene products. Curr Opin Genet Dev 1999, 9(1):22-30.

27. Shieh SY, Ikeda M, Taya Y, Prives C: DNA damage-induced phosphorylation of p53 alleviates inhibition by MDM2. Cell 1997, 91(3):325-334.

28. Siliciano JD, Canman CE, Taya Y, Sakaguchi K, Appella E, Kastan MB: DNA damage induces phosphorylation of the amino terminus of p53. Genes Dev 1997, 11(24):3471-3481. 
29. Suzuki M, Suzuki K, Kodama S, Yamashita S, Watanabe M: Persistent amplification of DNA damage signal involved in replicative senescence of normal human diploid fibroblasts. Oxid Med Cell Longev 2012, 2012:310534.

30. Zou HY, Li Q, Lee JH, Arango ME, McDonnell SR, Yamazaki S et al: An orally available small-molecule inhibitor of c-Met, PF-2341066, exhibits cytoreductive antitumor efficacy through antiproliferative and antiangiogenic mechanisms. Cancer Res 2007, 67(9):4408-4417.

31. Frink RE, Peyton M, Schiller JH, Gazdar AF, Shay JW, Minna JD: Telomerase inhibitor imetelstat has preclinical activity across the spectrum of non-small cell lung cancer oncogenotypes in a telomere length dependent manner. Oncotarget 2016, 7(22):31639-31651.

32. Heaphy CM, Subhawong AP, Hong SM, Goggins MG, Montgomery EA, Gabrielson E et al: Prevalence of the alternative lengthening of telomeres telomere maintenance mechanism in human cancer subtypes. Am J Pathol 2011, 179(4):1608-1615.

33. Efeyan A, Serrano M: p53: guardian of the genome and policeman of the oncogenes. Cell Cycle 2007, 6(9):1006-1010.

34. Brookes S, Rowe J, Ruas M, Llanos S, Clark PA, Lomax M et al: INK4a-deficient human diploid fibroblasts are resistant to RAS-induced senescence. Embo j 2002, 21(12):2936-2945.

35. Bromberg JF, Wrzeszczynska MH, Devgan G, Zhao Y, Pestell RG, Albanese C et al: Stat3 as an oncogene. Cell 1999, 98(3):295-303.

36. Dutta P, Sabri N, Li J, Li WX: Role of STAT3 in lung cancer. Jakstat 2014, 3(4):e999503.

37. Kojima H, Inoue T, Kunimoto H, Nakajima K: IL-6-STAT3 signaling and premature senescence. Jakstat 2013, 2(4):e25763.

38. Kojima H, Kunimoto H, Inoue T, Nakajima K: The STAT3-IGFBP5 axis is critical for IL-6/gp130induced premature senescence in human fibroblasts. Cell Cycle 2012, 11(4):730-739.

39. Koh J, Jang JY, Keam B, Kim S, Kim MY, Go H et al: EML4-ALK enhances programmed cell deathligand 1 expression in pulmonary adenocarcinoma via hypoxia-inducible factor (HIF)- 1 a and STAT3. Oncoimmunology 2016, 5(3):e1108514.

40. Tanizaki J, Okamoto I, Takezawa K, Sakai K, Azuma K, Kuwata K et al: Combined effect of ALK and MEK inhibitors in EML4-ALK-positive non-small-cell lung cancer cells. Br J Cancer 2012, 106(4):763767.

41. Cao Q, Li YY, He WF, Zhang ZZ, Zhou Q, Liu X et al: Interplay between microRNAs and the STAT3 signaling pathway in human cancers. Physiol Genomics 2013, 45(24):1206-1214.

42. Yoshida R, Sasaki T, Minami Y, Hibino Y, Okumura S, Sado M et al: Activation of Src signaling mediates acquired resistance to ALK inhibition in lung cancer. Int J Oncol 2017, 51(5):1533-1540.

43. Kandhaya-Pillai R, Miro-Mur F, Alijotas-Reig J, Tchkonia T, Kirkland JL, Schwartz S: TNFalphasenescence initiates a STAT-dependent positive feedback loop, leading to a sustained interferon signature, DNA damage, and cytokine secretion. Aging (Albany NY) 2017, 9(11):2411-2435. 
44. Chen X, Xie ZH, Lv YX, Tang QP, Zhang H, Zhang JY et al: A proteomics analysis reveals that A2M might be regulated by STAT3 in persistent allergic rhinitis. Clin Exp Allergy 2016, 46(6):813-824.

45. Dauer DJ, Ferraro B, Song L, Yu B, Mora L, Buettner R et al: Stat3 regulates genes common to both wound healing and cancer. Oncogene 2005, 24(21):3397-3408.

46. De Giglio A, Porreca R, Brambilla M, Metro G, Prosperi E, Bellezza G et al: Fatal acute disseminated intravascular coagulation as presentation of advanced ALK-positive non-small cell lung cancer: Does oncogene addiction matter? Thromb Res 2018, 163:51-53.

47. Fofaria NM, Srivastava SK: STAT3 induces anoikis resistance, promotes cell invasion and metastatic potential in pancreatic cancer cells. Carcinogenesis 2015, 36(1):142-150.

48. Guadamillas MC, Cerezo A, Del Pozo MA: Overcoming anoikis-pathways to anchorage-independent growth in cancer. J Cell Sci 2011, 124(Pt 19):3189-3197.

49. Mallette FA, Gaumont-Leclerc MF, Ferbeyre G: The DNA damage signaling pathway is a critical mediator of oncogene-induced senescence. Genes Dev 2007, 21(1):43-48.

50. Di Micco R, Fumagalli M, Cicalese A, Piccinin S, Gasparini P, Luise C et al: Oncogene-induced senescence is a DNA damage response triggered by DNA hyper-replication. Nature 2006, 444(7119):638-642.

51. Bartkova J, Rezaei N, Liontos M, Karakaidos P, Kletsas D, Issaeva N et al: Oncogene-induced senescence is part of the tumorigenesis barrier imposed by DNA damage checkpoints. Nature 2006, 444(7119):633-637.

52. Sperka T, Wang J, Rudolph KL: DNA damage checkpoints in stem cells, ageing and cancer. Nat Rev Mol Cell Biol 2012, 13(9):579-590.

53. Martinez P, Blasco MA: Telomeric and extra-telomeric roles for telomerase and the telomere-binding proteins. Nat Rev Cancer 2011, 11(3):161-176.

54. Saretzki G: Extra-telomeric functions of human telomerase: cancer, mitochondria and oxidative stress. Curr Pharm Des 2014, 20(41):6386-6403.

55. Farwell DG, Shera KA, Koop JI, Bonnet GA, Matthews CP, Reuther GW et al: Genetic and epigenetic changes in human epithelial cells immortalized by telomerase. Am J Pathol 2000, 156(5):1537-1547.

56. Morales CP, Holt SE, Ouellette M, Kaur KJ, Yan Y, Wilson KS et al: Absence of cancer-associated changes in human fibroblasts immortalized with telomerase. Nat Genet 1999, 21(1):115-118.

57. Lee JJ, Park S, Park H, Kim S, Lee J, Lee J et al: Tracing Oncogene Rearrangements in the Mutational History of Lung Adenocarcinoma. Cel/2019, 177(7):1842-1857.

58. Sedelnikova OA, Horikawa I, Zimonjic DB, Popescu NC, Bonner WM, Barrett JC: Senescing human cells and ageing mice accumulate DNA lesions with unrepairable double-strand breaks. Nat Cell Biol 2004, 6(2):168-170.

\section{Figures}


Fig. 1

a

$$
\text { Dox: } \frac{\text { CRL-2097/TR }}{-\quad-\quad-\quad+}
$$

Transduction: Vec EML4-ALK

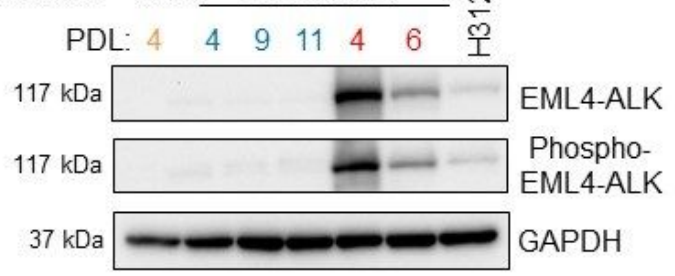

b

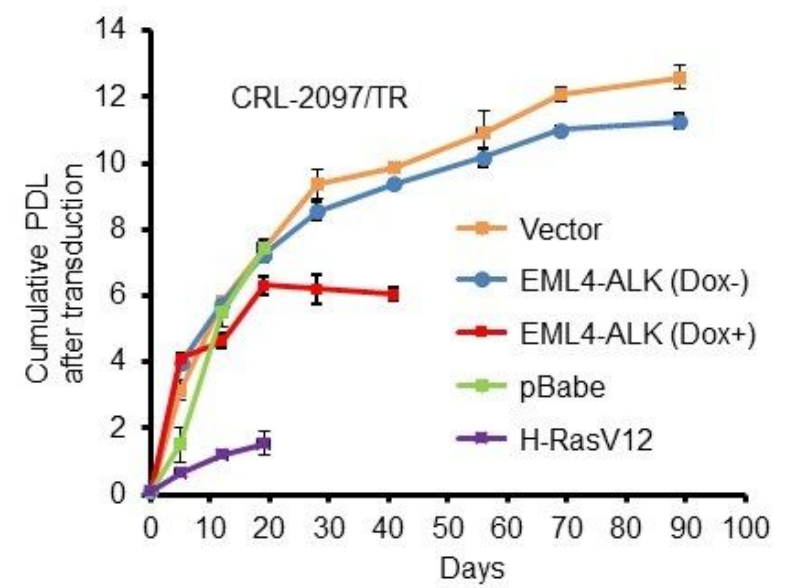

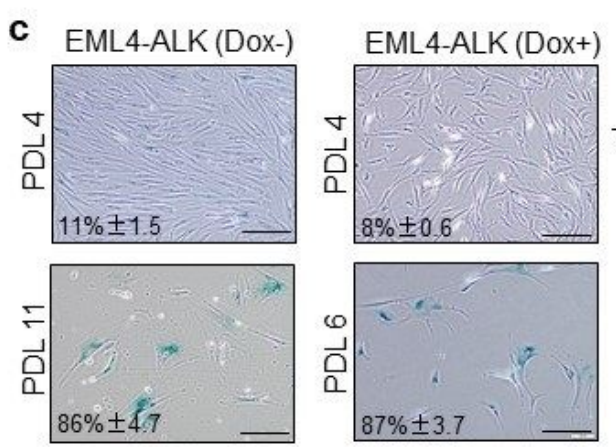

(Fig. 1. Continued)
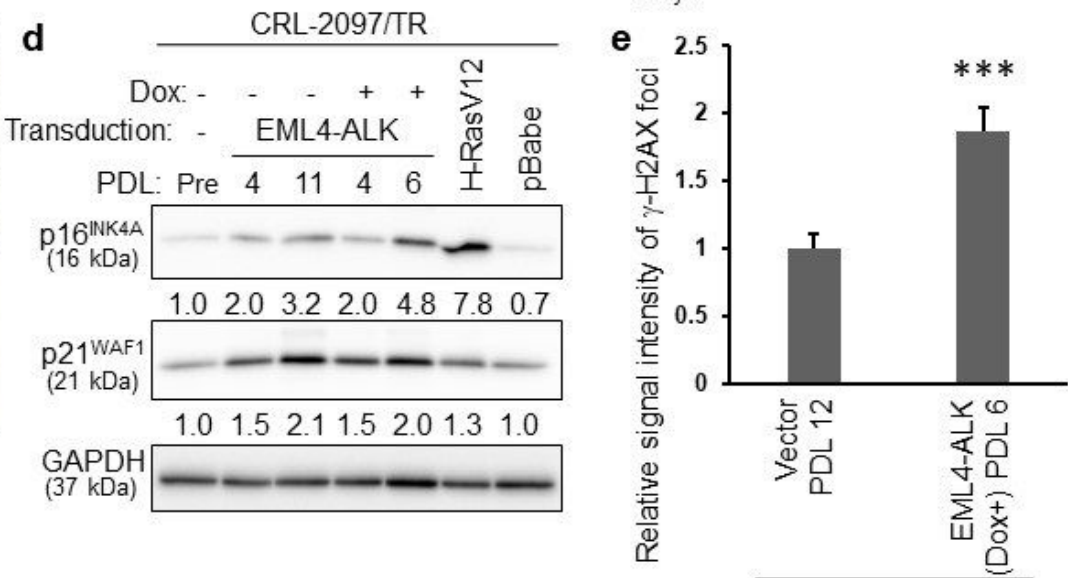

CRL-2097/TR

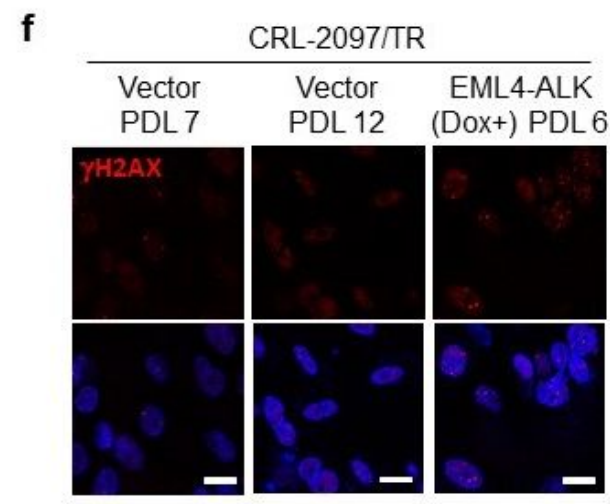

\section{Figure 1}

EML4-ALK induces early onset of cellular senescence in mortal normal human fibroblasts. CRL-2097/TR were transduced with an inducible lentiviral vector of EML4-ALK or its control vector (Vec). Shown PDL is after transduction. (a) Western blot analysis confirming the inducible expression of EML4-ALK. The EML4-ALK- or Vec-transduced cells with (+) or without (-) doxycycline induction (Dox) were examined at indicated PDL for protein expression levels of total EML4-ALK and phosphorylated EML4-ALK. GAPDH 
(glyceraldehyde 3-phosphate dehydrogenase) was a loading control. H3122 (EML4-ALK expressing cells). Full-length blots are presented in Supplementary Figure. (b) Replicative lifespan of transduced CRL2097/TR fibroblasts. Cumulative PDL after transduction were plotted to days after transduction. According to ATCC's PDL counting, CRL-2097/TR at the initiation of this experiment corresponded to PDL 43 in total. The proliferation arrest of Vector or Dox (-) control cells at PDL 11 or 12 after transduction, corresponding to PDL 54 or 55 in total, is consistent with the information from ATCC that this strain senesces at PDL 56. (c) SA- $\beta$-gal staining of CRL-2097/TR fibroblasts with (Dox+) and without (Dox-) induced expression of EML4-ALK. Representative images in proliferative phase (both at PDL 4) and growth arrested phase (Dox+ at PDL 6 and Dox- at PDL 11) are shown. Percentages of SA- $\beta$-gal positive cells (means \pm s.d.) were from biological triplicate, in each of which more than 300 cells were observed. Scale bars, $20 \mu \mathrm{m}$. (d) Western blot analysis of p16INK4A and p21WAF1. The EML4-ALK-transduced CRL2097/TR fibroblasts with (+) and without (-) Dox induction at indicated PDL, in parallel to the cells before transduction (Pre), and those retrovirally transduced with $\mathrm{H}$-RasV12 and its control vector pBabe (Supplementary Fig. S1A-B) were examined. Quantitative expression levels of p16INK4A and p21WAF1 (normalized with GAPDH) are shown in relative to the levels in the cells before transduction (Pre). (e) Early and accelerated accumulation of DNA damage in cells expressing EML4-ALK. Senescent CRL2097/TR with control vector (PDL 12) and those with Dox-induced EML4-ALK expression (PDL 6) were examined for $\mathbb{\nabla}-\mathrm{H} 2 \mathrm{AX}$ foci. The data (signal intensity per cell) are mean \pm s.d. from triplicates and shown relative to the vector control. (f) Representative images of $\mathbb{X}-\mathrm{H} 2 \mathrm{AX}$ immunofluorescence staining in the cells shown in (e). The lower panels were merged with DAPI. CRL-2097/TR with control vector (PDL 7) is also shown as comparison. Note that the level of $\mathbb{B}-\mathrm{H} 2 \mathrm{AX}$ foci in natural replicative senescence (Vector, PDL 12) is consistent with that previously reported [58]. Scale bars, $20 \mu \mathrm{m}$. 
Fig. 2
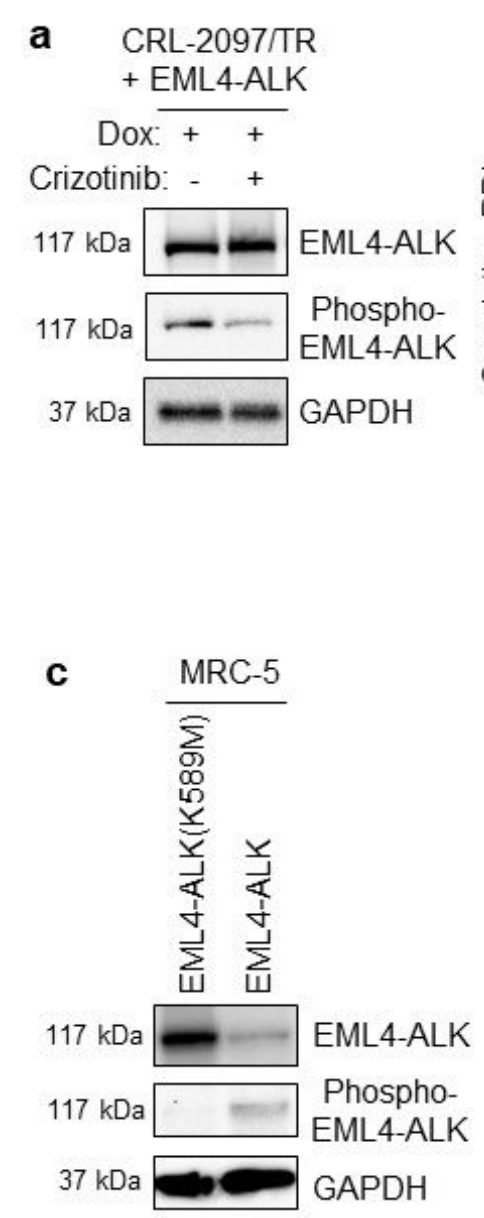
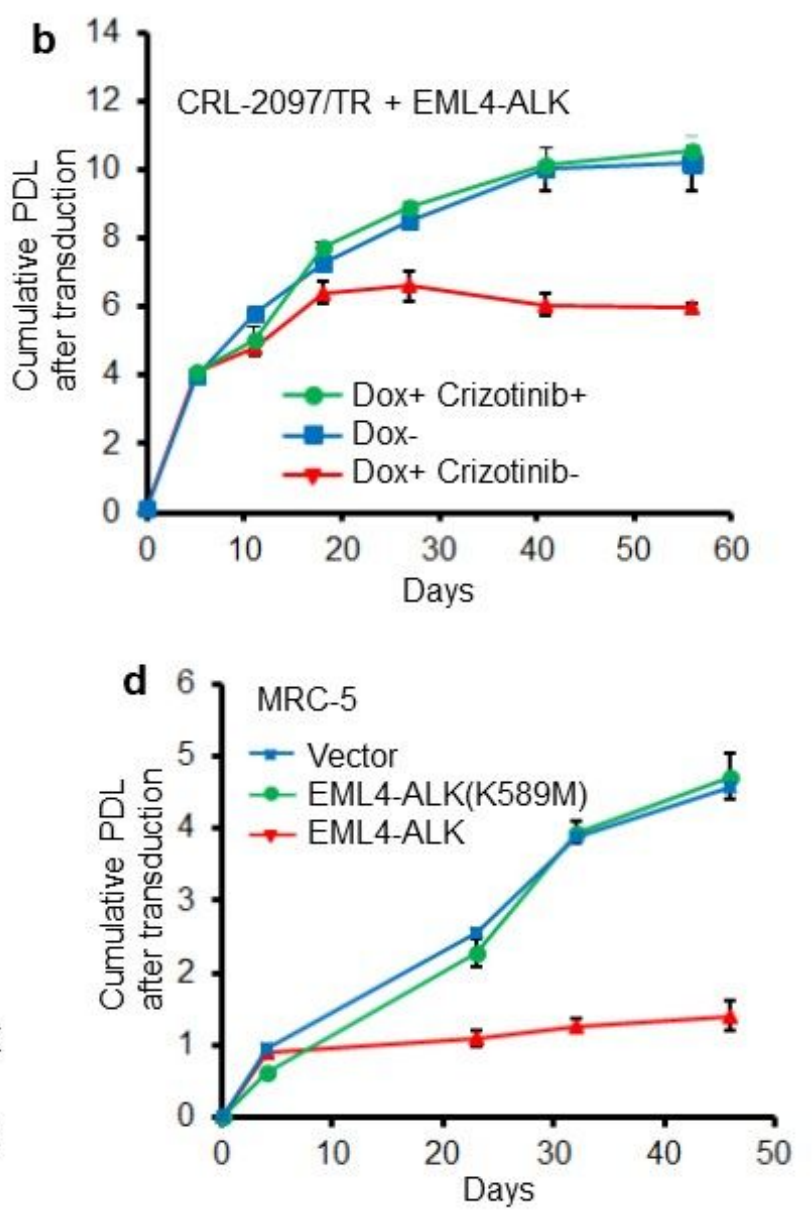
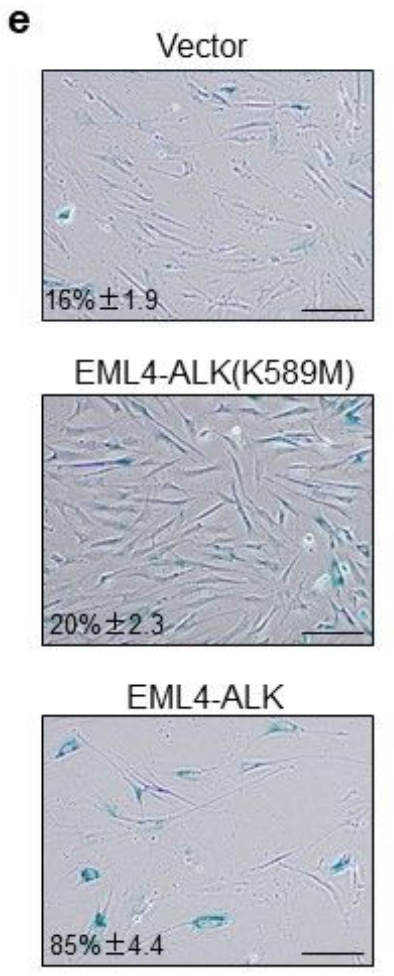

\section{Figure 2}

The kinase activity of EML4-ALK is required for early induction of cellular senescence. (a) Western blot analysis showing a decrease in EML4-ALK autophosphorylation by an ALK TKI Crizotinib. CRL-2097/TR fibroblasts with Dox-induced expression of EML4-ALK were maintained in culture in the absence (-) or presence (+) of $25 \mathrm{nM}$ Crizotinib and examined for levels of total EML4-ALK (top) and phosphorylated EML4-ALK (middle). Dox was added at day 0 and Crizotinib was added at day 5 and both remained included throughout the experiment with medium change every 48 hours. The cells were harvested at day 40 (at PDL 6 or 10) for western blot. (b) Abrogation of early senescence by Crizotinib. The Dox-treated cells (Dox + ) with and without Crizotinib ( + and -) shown in (a), along with the untreated cells (Dox-), were examined for cumulative PDL after transduction as in Fig. 1b. (c) Western blot analysis of MRC-5 fibroblasts that express wild-type EML4-ALK and the kinase-dead mutant (K589M). MRC-5, a second strain of mortal normal human fibroblasts, were transduced with the lentiviral vector of EML4-ALK (the same vector as used above, which drives constitutive expression in the absence of a Tet repressor) and its K589M mutant derivative. Western blot analysis was performed as in (a). (d) Dependence of early senescence on the kinase activity of EML4-ALK. The transduced MRC-5 fibroblasts shown in (c), along 
with the vector control-transduced cells, were examined for cumulative PDL after transduction, as in (b) and Fig. 1b. Note that this experiment used late-passage MRC-5 with fewer PDL remaining until natural replicative senescence, compared with CRL-2097/TR used in the above experiments. (e) Representative images of SA- $\beta$-gal staining with quantitative data of positive cells (mean \pm s.d., as in Fig. 1c). The cells shown in (d) were examined at day 22. Scale bars, $20 \mu \mathrm{m}$.

Fig. 3
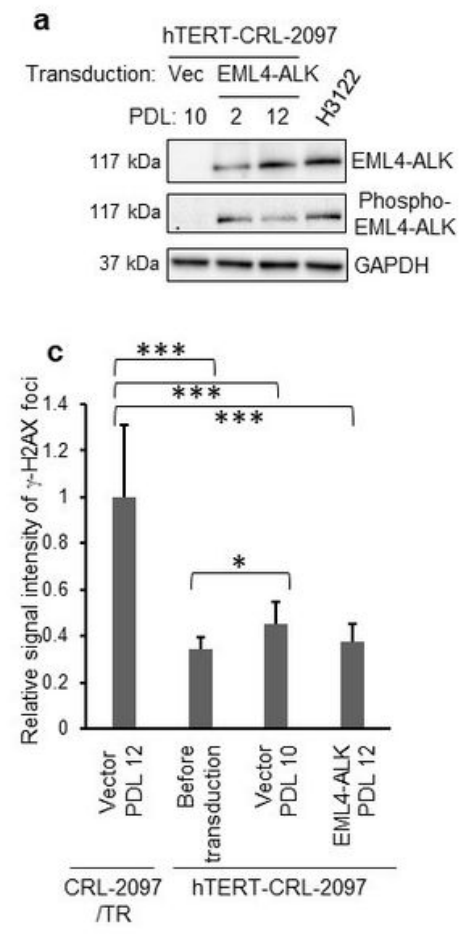

(Fig. 3. Continued)

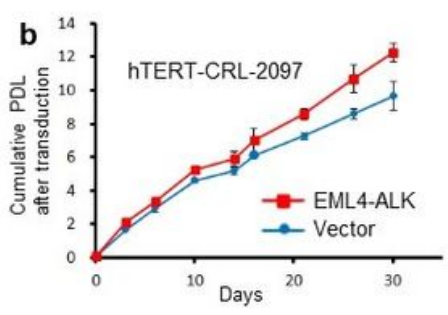

$$
\text { d }
$$

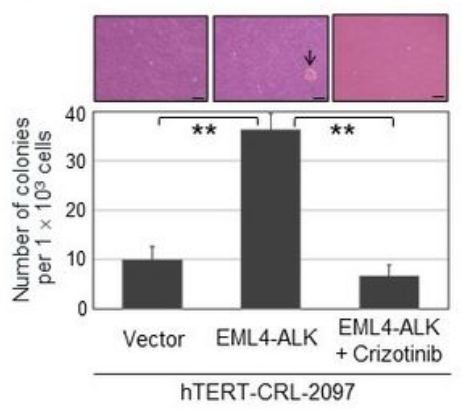

\begin{tabular}{|l|l|l|l|}
\hline Cells & Karyotype & TP53 & Codon 72 \\
\hline hTERT-CRL-2097 & $46, \mathrm{XY}$ & Wild-type & Pro/Arg \\
\hline hTERT-CRL-2097 +Vector & $46, \mathrm{XY}$ & n.d & n.d \\
\hline hTERT-CRL-2097 +EML4-ALK & $46, \mathrm{XY}$ & Wild-type & Pro/Arg \\
\hline Soft-agar clone \#1 & $46, \mathrm{XY}$ & Wild-type & Pro/Arg \\
\hline Soft-agar clone \#2 & $46, \mathrm{XY}$ & Wild-type & Pro/Arg \\
\hline Soft-agar clone \#3 & $46, \mathrm{XY}$ & Wild-type & Pro/Arg \\
\hline Soft-agar clone \#4 & $46, \mathrm{XY}$ & Wild-type & Pro/Arg \\
\hline Soft-agar clone \#5 & $46, \mathrm{XY}$ & Wild-type & Pro/Arg \\
\hline Soft-agar clone \#6 & $46, \mathrm{XY}$ & Wild-type & Pro/Arg \\
\hline
\end{tabular}

\section{Figure 3}

EML4-ALK promotes anchorage-independent growth in hTERT-transduced normal human fibroblasts. (a) hTERT-CRL-2097 were transduced with the EML4-ALK lentiviral vector (for the constitutive expression in the absence of a Tet repressor) or the control vector (Vec). Western blot analysis was performed in the cells at indicated PDL after transduction, as in Fig. 1a, with H3122 as a positive control. (b) Cell proliferation curves of hTERT-CRL-2097 with the expression of EML4-ALK or the control vector. Cumulative PDL were calculated and plotted to days after transduction, as above. (c) No accumulation of DNA damage by EML4-ALK in hTERT-transduced fibroblasts. The hTERT-CRL-2097 cells expressing EML4-ALK (PDL 12), with the control vector (PDL 10) and before transduction were examined for $\mathbb{B}-\mathrm{H} 2 \mathrm{AX}$ foci as in Fig. 1e. The CRL-2097/TR fibroblasts at senescence (Vector at PDL 12 in Fig. 1b and 1e) were again examined for comparison and shown in parallel. The data quantification and analysis were as in Fig. 1e. ${ }^{*} P<0.05$; ${ }^{\star \star *} P<0.001$. (d) Anchorage-independent growth of hTERT-CRL-2097 cells expressing EML4-ALK, with and without $25 \mathrm{nM}$ Crizotinib. Those cells with the control vector were plated in soft-agar medium and examined for colony formation at day 21. Numbers of colonies per $1 \otimes 103$ cells plated (means \pm s.d. from biological triplicate) are shown with representative images without or with a colony 
(arrow). Scale bars, $50 \mu \mathrm{m}$. ${ }^{\star} \mathrm{P}<0.01$. (e) Summary of karyotype and the status of the TP53 gene in EML4-ALK-expressing hTERT-CRL-2097 fibroblasts and their derived cell clones isolated and established from soft-agar colonies (\#1 to \#6). n.d., not determined.

Fig. 4

a

CRL:-2097/TR

Transduction: Vec EML4-ALK

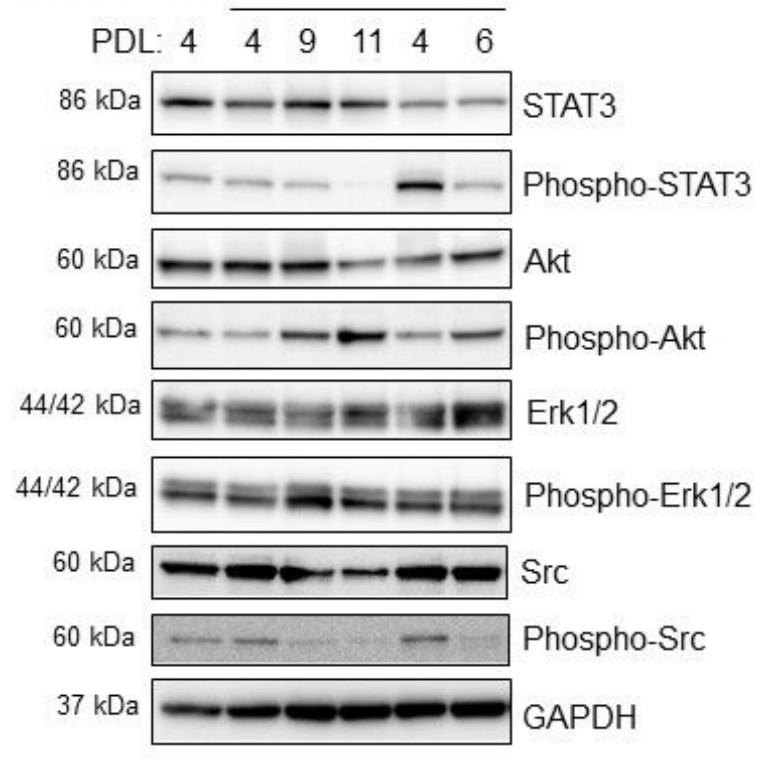

b

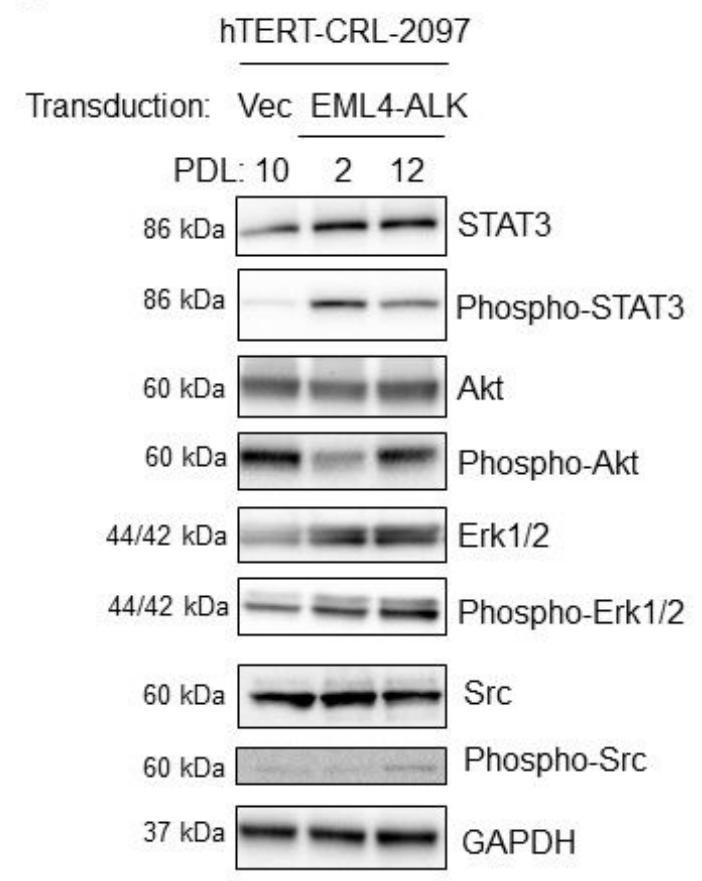

\section{Figure 4}

STAT3 is phosphorylated by EML4-ALK in both mortal and hTERT-transduced normal human fibroblasts. The same set of CRL-2097/TR-derived cells as in Fig. 1a (a) and the same set of hTERT-CRL-2097-derived cells as in Fig. 3a (b) were examined in western blot analysis for levels of total and phosphorylated STAT3, total and phosphorylated Akt, total and phosphorylated Erk1/2, and total and phosphorylated Src. Full-length blots/gels are presented in Supplementary Figure. 
Fig. 5
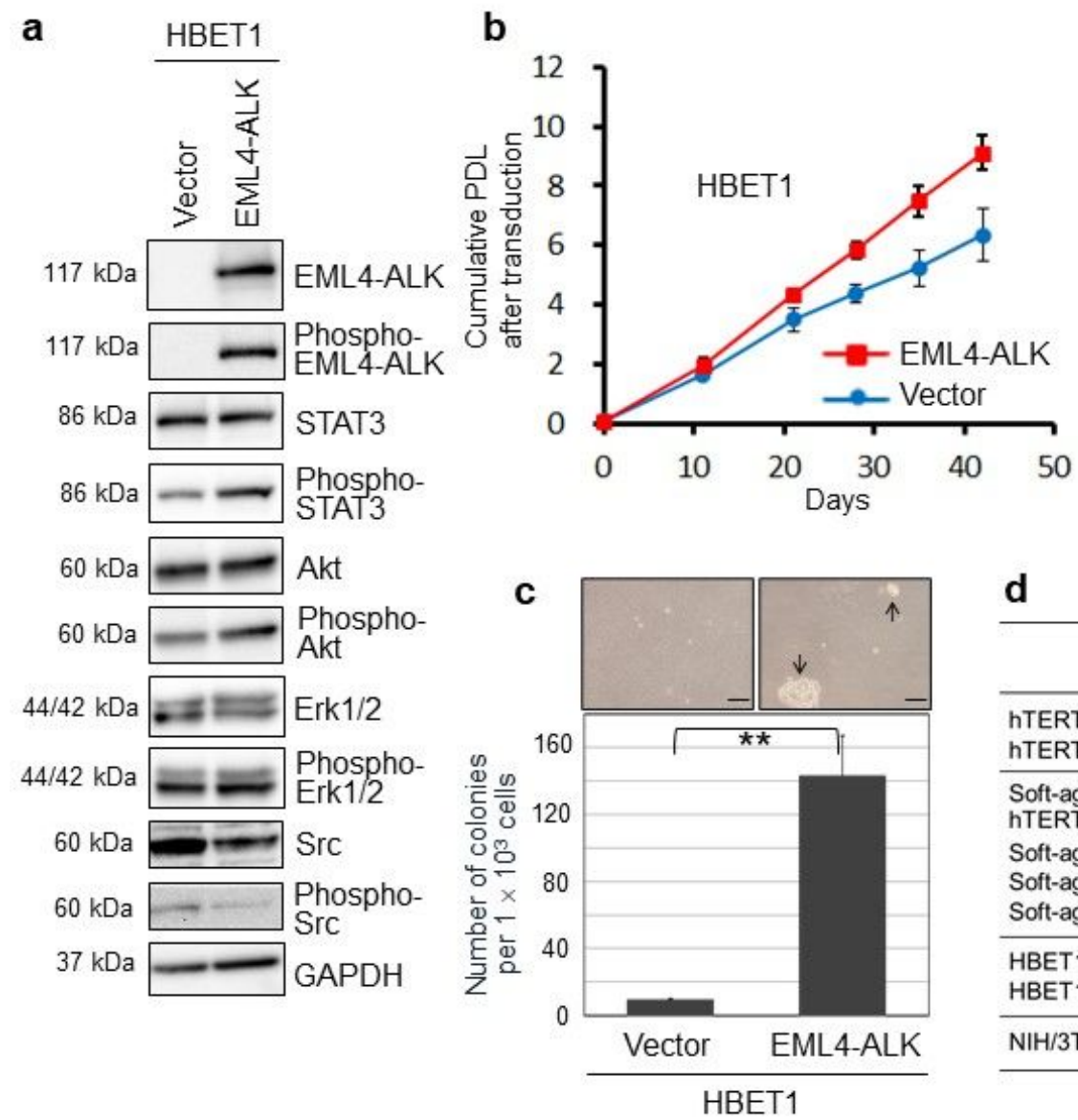

d

\begin{tabular}{lcc}
\hline \multicolumn{1}{c}{ Cells } & $\begin{array}{c}\text { Number of } \\
\text { injected sites }\end{array}$ & $\begin{array}{c}\text { Number of } \\
\text { tumors formed }\end{array}$ \\
\hline hTERT-CRL-2097+Vector & 25 & 0 \\
hTERT-CRL-2097+EML4-ALK & 10 & 0 \\
\hline Soft-agar clones derived from & & \\
hTERT-CRL-2097+EML4-ALK & 5 & 0 \\
Soft-agar clone \#1 & 5 & 0 \\
Soft-agar clone \#3 & 5 & 0 \\
Soft-agar clone \#4 & 10 & 0 \\
\hline HBET1+Vector & 10 & 0 \\
HBET1+EML4-ALK & 4 & 4 \\
\hline NIH/3T3+EML4-ALK (positive control) & 4 & \\
\hline
\end{tabular}

\section{Figure 5}

EML4-ALK accelerates cell proliferation and promotes anchorage-independent growth in hTERTimmortalized normal human bronchial epithelial cells. (a) HBET1, an hTERT-immortalized normal human bronchial epithelial cell line, was transduced with the EML4-ALK lentiviral vector or the control vector as in Fig. 3. The cells at 28 days after transduction were examined in western blot analysis as in Fig. 3a and Fig. 4. Full-length blots/gels are presented in Supplementary Figure. (b) Cell proliferation curves of HBET1 expressing EML4-ALK or with the control vector. Cumulative PDL were calculated and plotted to days after transduction, as above. (c) Anchorage-independent growth of HBET1 cells expressing EML4-ALK. The HBET1 cells with EML4-ALK or the control vector were examined for anchorage-independent growth, as in Fig. 3d and S5C. Data analysis and presentation are also as in Fig. 3d and S5C. Arrows in a representative image indicate colonies that formed in soft-agar medium. Scale bars, $50 \mu \mathrm{m}$. ${ }^{*} \mathrm{P}<0.01$. (d) Summary of tumorigenicity assay in NOD.SCID/Ncr mice. Cells were subcutaneously injected into each flank ( 5 \& 106 per flank) of mice at 6-10 weeks of age, followed by observation until 8 weeks after injection. In each mouse, one flank had EML4-ALK-expressing cells or soft-agar clones, and the other flank had cells with the control vector. Whereas NIH/3T3 cells expressing EML4-ALK (2 $\otimes 106$ per flank, at 
both flanks of two mice) produced tumors necessitating euthanasia, no progressively growing tumors formed from any of hTERT-CRL-2097- and HBET1-derived cells.

\section{Supplementary Files}

This is a list of supplementary files associated with this preprint. Click to download.

- Table1.xlsx

- ARRIVEChecklistOriginal.pdf

- ARRIVEChecklistPlainversion.pdf

- SupplementaryFigandTable.pdf 